\title{
Failure of ipratropium bromide to modify the diurnal variation of asthma in asthmatic children
}

\author{
PETER D SLY, LOUIS I LANDAU, ANTHONY OLINSKY \\ From the Professorial Department of Thoracic Medicine and Department of Paediatrics, Royal Children's \\ Hospital, Melbourne, and University of Melbourne, Melbourne, Australia
}

ABSTRACT Thirty one children with asthma were given $40 \mu \mathrm{g}$ of ipratropium bromide and identical placebo by inhalation three times a day in a double blind, randomised crossover study to test the ability of an anticholinergic drug to modify the diurnal variation in airway calibre and bronchial reactivity. Subjects measured peak expiratory flow rate approximately eight hourly, before and after inhaled salbutamol, for four week periods. Paired $t$ tests and cosinor analysis were used to assess the diurnal variation in airway calibre from the peak expiratory flow rate recorded before salbutamol and to assess the diurnal variation in bronchodilator responsiveness from the increase in peak expiratory flow rate after salbutamol. Maintenance treatment with ipratropium bromide $40 \mu \mathrm{g}$ three times daily reduced the provocative dose of histamine which caused a $20 \%$ fall in $\mathrm{FEV}_{1}$ (geometric mean $\left.\mathrm{PD}_{20}=0.78 v 0.49 \mathrm{mg} / \mathrm{ml}, \mathrm{p}<0.05\right)$, despite an eight to 12 hour gap between the last dose of ipratropium and histamine challenge. It did not, however, diminish the diurnal variation in airway calibre (mean amplitude $=12.7 v 10.1$ ) or in bronchodilator responsiveness (mean amplitude $=62.4 v 63.5$ ). There was no improvement in the clinical state of subjects while they were taking ipratropium bromide.

A diurnal variation in airway calibre, as judged by peak expiratory flow (PEF), has been described in adults with asthma ${ }^{1}$ and children with asthma. ${ }^{23}$ The mechanisms underlying this rhythm are unknown. Diurnal variation in airway sensitivity to histamine 4 and to acetylcholine ${ }^{5}$ has also been described and could not be explained by variation in baseline airway calibre. The mechanisms underlying this diurnal variation in airway sensitivity are not known. Bronchoconstriction after inhalation of histamine is thought to be mediated in part by vagal reflexes. ${ }^{6}$ Thus the diurnal variation in airway sensitivity to histamine, and other substances, may also be mediated in part through the vagus.

Nocturnal exacerbations of asthma have been attributed to an exaggeration of the diurnal variation

Address for reprint requests: Dr PD Sly, Meakins-Christie Laboratories, McGill University, 3775 University Street, Montreal, Quebec H3A 2B4, Canada.

Accepted 28 August 1986 in airway calibre, ${ }^{1}$ but the heightened airway sensitivity seen in the early hours of the morning ${ }^{4} 5$ may also be a contributing factor. If nocturnal asthma is related to the diurnal variation in airway calibre or in bronchial reactivity, or both, a treatment regimen that would abolish or diminish these variations might be able to decrease some of the morbidity and mortality associated with nocturnal asthma. ${ }^{78}$ In recent studies anticholingergic drugs have been shown to increase early morning PEF more than evening PEF $^{9}$ and to decrease morning dipping, ${ }^{10}$ which suggests that these agents may be able to diminish the diurnal variation in airway calibre.

This study was performed to test the ability of the anticholinergic agent ipratropium bromide in clinically acceptable doses to modify the diurnal variations in airway calibre and in bronchodilator responsiveness. Thirty one children with asthma were given ipratropium bromide and placebo in a double blind crossover study. The diurnal variation in airway calibre was assessed from PEF recordings and the diurnal variation in bronchodilator responsiveness assessed from the increase in PEF after salbutamol. 


\section{Methods}

Thirty one children with asthma ( 21 male, 10 female), aged from 8 to 18 years (mean 12.0 years), were studied. The study consisted of four periods of four weeks: a baseline period and two treatment periods separated by a washout period. Each child kept a diary card recording a daily symptom score, medications taken, and PEF (Wright mini peak flow meter) approximately eight hourly, before and 10 minutes after inhaling salbutamol from an aerosol. PEF was measured on waking in the morning, at 1600 hours, and at bed time, as suggested by Hetzel and Clark. ${ }^{1}$ At each test time the children carried out three measurements and recorded the highest PEF on the diary card. Children were maintained on their usual medication and carried out normal activities during the study. Medications used were: salbutamol, two puffs from a metered dose aerosol $(200 \mu \mathrm{g})$ or 0.03 $\mathrm{ml} / \mathrm{kg}$ up to a maximum of $1.0 \mathrm{ml}$ of a $0.5 \%$ solution by wet nebulisation; a 12 hour slow release theophylline preparation at a dose of $20 \mathrm{mg} / \mathrm{kg}$ per day to a maximum dose of $500 \mathrm{mg}$ twice daily or as directed by blood concentrations; and inhaled beclomethasone dipropionate by a metered dose aerosol, $50 \mu \mathrm{g}$ per puff, two to four puffs three or four times daily.

Each day the children were required to give a score between zero and three for each of the following: amount of wheeze during the night; amount of cough during the night; amount of wheeze during the day; and limitation of activity during the day. Symptom scores were added to give a daily total for each child. Daily symptom scores could range from zero, for an asymptomatic day, up to 12 for a day with a pronounced cough, wheeze, and limitation of activity. Daily symptom scores were added to give a symptom score for each period for each child. The study treatment consisted of two puffs of either ipratropium bromide $(40 \mu \mathrm{g})$ or placebo three times a day from identical metered dose aerosols labelled A or B. The children were randomly allocated to receive either aerosol A or B first.

Histamine challenge tests were performed, following the protocol of Yan et al, ${ }^{11}$ at the same time of day, at the completion of each study period. Subjects did not use salbutamol or treatment aerosols for between eight and 12 hours before the challenge test. Other medications were continued, but as subjects were taking the same treatment regimen throughout the study this should not contribute to any differences found between the challenge tests. Dose-response curves were plotted and the cumulative dose of histamine causing a $20 \%$ fall in $\mathrm{FEV}_{1}\left(\mathrm{PD}_{20}\right)$ calculated.

The study protocol was approved by the hospital's ethics committee, and informed consent was obtained from each subject and at least one parent.

DATA ANALYSIS

All statistical tests were performed on natural log transformed $\mathrm{PD}_{20}$ data. Wilcoxon's matched pairs signed ranks test was used to compare $\mathrm{PD}_{20}$ value between study periods. Dunnett's paired $t$ test $^{12}$ was used to test differences between afternoon, morning and evening PEF values for each child within each. period, afternoon PEF being considered as "control ${ }^{\odot}$ and morning and evening PEF as two "treatments. $\frac{\vec{\omega}}{\omega}$

The amplitude of the diurnal variation in PEF was assessed by cosinor analysis, using the modified tech $\vec{x}$ nique reported by Hetzel and Clark. ${ }^{1}$ PEF was anal ysed against time using the equation:

$\mathrm{PEF}=C o+a \cos (2 \pi t / 24)+b \sin (2 \pi t / 24)$, where $C o=$ mean value or intercept; $t=$ time PEE was measured. Coefficients $a$ and $b$ were determine and used to calculate the "peak to trough" amplitude $\left(A=2 \sqrt{a^{2}+b^{2}}\right)$. Amplitude was expressed as percentage of the subject's mean PEF for the period. under study, and compared between treatment perbo ods by paired $t$ test.

The increase in PEF $(1 / \mathrm{min})$ after salbutame 8 inhalation was calculated at each test time. Brons chodilator responsiveness was analysed as the absō lute increase in PEF as a function of time by cosinoü analysis, as described above. Amplitude, expressed $\frac{\mathbb{Q}}{\mathrm{a}}$ a percentage of the subject's mean increase in PEE after salbutamol inhalation for the period undes study, was compared between treatment periods bु paired $t$ test. Dunnett's paired $t$ test $^{12}$ was used to compare the increase in PEF after salbutamol at each test time on data for each child.

Symptom scores and excess bronchodilator med cation were compared between treatment periods bo paired $t$ tests. The null hypothesis, that there were no differences between ipratropium bromide and placebo, was rejected at $\mathrm{p}<0.05$.

\section{Results}

Treatment with ipratropium bromide caused n change in mean PEF (table 1). Eight children hajb higher morning PEF values while taking ipratropiu bromide, but another eight had higher morning PEF values while taking placebo.

\section{HISTAMINE CHALLENGE}

Treatment with ipratropium bromide was associated with significantly higher mean $\mathrm{PD}_{20}$ values (ge metric mean $0.78 v 0.49 \mathrm{mg} / \mathrm{ml}, \mathrm{p}<0.01$ ) (figure).

DIURNAL VARIATION IN AIRWAY CALIBRE Seventy four per cent ( 23 of 31 ) of subjects showed 
Table 1 Group mean peak flow rates

\begin{tabular}{ll} 
& Mean (SD) \\
\hline Baseline & \\
Morning & $309(82)$ \\
Afternoon & $332(79)$ \\
Evening & $320(81)$ \\
Ipratropium bromide & \\
Morning & $305(94)$ \\
Afternoon & $325(89)$ \\
Evening & $325(90)$ \\
Placebo & \\
Morning & \\
Afternoon & $309(92)$ \\
Evening & $325(85)$ \\
\hline
\end{tabular}

significant diurnal variation (Dunnett's paired $t$ test) during the baseline period. The mean (SD) amplitude of the diurnal variation in PEF was $23 \cdot 1 \%(13 \cdot 7 \%)$. Treatment with ipratropium bromide did not affect the diurnal variation in PEF. Table 1 shows group mean PEF data for each treatment period. Seventy seven per cent ( 24 of 31 ) of subjects had significant

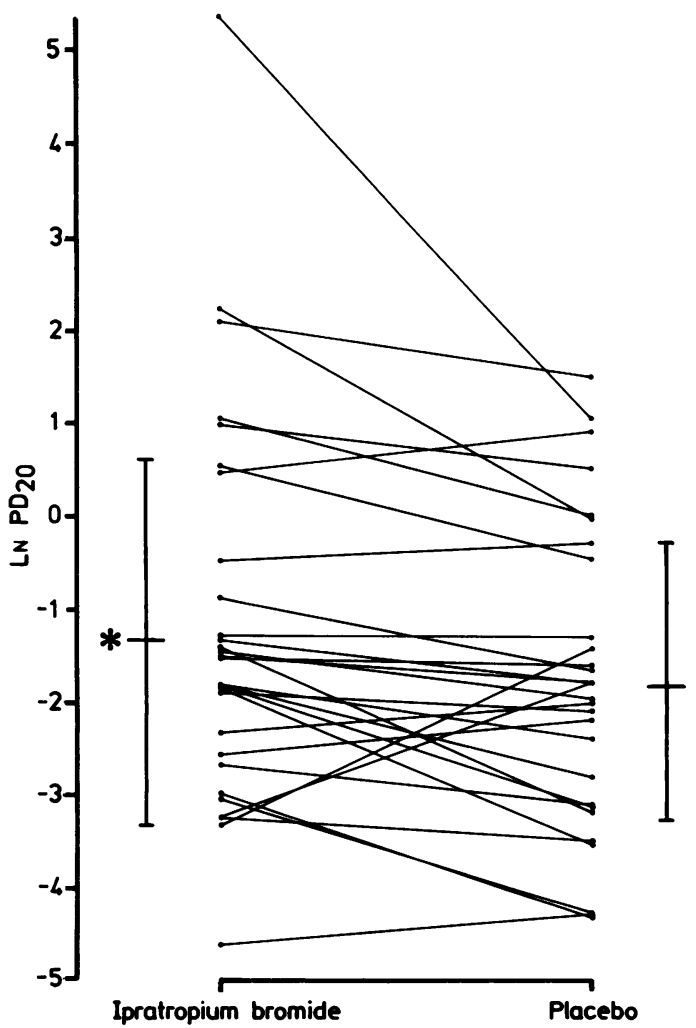

Results of histamine challenge tests (In $P D_{20}$ ) after the ipratropium bromide and placebo periods for each subject; $p<0.05$. diurnal variations while taking ipratropium bromide compared with $68 \%$ (21 of 31$)$ taking placebo. When the eight children who had higher morning PEF recordings were examined separately there was no relation between the increase in PEF and the diurnal variation in PEF. There was no difference between the mean (SD) amplitude of the diurnal variation in PEF on ipratropium bromide (mean $22.9 \%(13.7)$ ) and placebo (mean $22 \cdot 1 \%,(16 \cdot 2)$ ).

\section{DIURNAL VARIATION IN BRONCHODILATOR RESPONSIVENESS}

Twenty one children inhaled salbutamol in constant dosage, regularly three times a day. Fifty seven per cent (12 of 21) had significantly greater bronchodilatation in the morning during both ipratropium bromide and placebo periods $(p<0.05)$. Treatment with ipratropium bromide did not alter the amplitude of the diurnal variation in bronchodilator responsiveness (mean amplitude $=63.5 \%$ (range $13-138 \%$ ) during placebo period; $62.4 \%$ (range $12-145 \%$ ) during ipratropium bromide period). The absolute increases in PEF after salbutamol were not altered by treatment with ipratropium bromide. Mean (SD) increases in PEF $(\mathrm{l} / \mathrm{min})$ for the morning, afternoon, and evening were $36(19 \cdot 7), 22(9 \cdot 7)$, and $29(13.5)$ respectively for the placebo period, and 34 (19.2), $22(11.9)$, and $29(16 \cdot 2)$ respectively for the ipratropium bromide period.

\section{DIARY CARDS}

There were no differences in symptom scores or additional doses of salbutamol during the baseline, ipratropium bromide, or placebo periods (table 2). No child was able to reduce maintenance treatment while taking ipratropium bromide.

\section{Discussion}

Treatment with ipratropium bromide $(40 \mu \mathrm{g})$ caused no change in mean PEF values in the 31 children who took part in this study, and it failed to modify the diurnal variation in airway calibre or in bron-. chodilator responsiveness to salbutamol. Nor did it reduce symptom scores or decrease the use of additional doses of salbutamol.

Table 2 Diary card information

\begin{tabular}{lll}
\hline & Symptom scores & $\begin{array}{l}\text { Additional doses } \\
\text { of salbutamol }\end{array}$ \\
\hline Baseline & $35(34 \cdot 2)$ & $14(22 \cdot 1)$ \\
Ipratropium bromide & $37(46 \cdot 7)$ & $19(35 \cdot 3)$ \\
Placebo & $32(51 \cdot 0)$ & $13(29 \cdot 1)$ \\
\hline
\end{tabular}

Results are group means (SD) of the total reported for each study period by each subject. 
In previous reports it has been suggested that ipratropium bromide may decrease the diurnal variation in PEF. ${ }^{910}$ The results of this study do not support this suggestion. The reasons for the difference between the results of this study and those in previous reports are not obvious. Mann and Hiller, ${ }^{9}$ who also studied children, used the same dosage regimen of ipratropium bromide as we did, and measurements were also made three times a day. They did not examine the diurnal variation in PEF directly but they found significantly higher mean PEF values in the morning with ipratropium bromide. Evening PEF recordings were also higher with ipratropium bromide but the increase was not significant. In the present study the effect of ipratropium bromide was assessed in each subject by comparing morning and afternoon PEF values each day using Dunnett's paired $t$ test. Mann and Hiller ${ }^{9}$ appear to have used a paired $t$ test on the group as a whole, comparing only the mean value of morning and evening PEF for each subject. In the present study eight children had significantly higher morning PEF values when taking ipratropium but only four of these had lower amplitudes of the diurnal variation in PEF while taking ipratropium. This suggests that simply comparing mean morning and evening (or afternoon) PEF values on and off a treatment for a group as a whole may not be the best way to evaluate the effect of that medication on the diurnal variation in PEF.

Maintenance treatment with clinically acceptable doses of ipratropium bromide did not result in clinical improvement in the children in this study. Most reported symptoms of asthma during the study and most required additional doses of salbutamol. Ipratropium bromide treatment did not reduce symptom scores or result in the use of fewer additional doses of salbutamol. These results, coupled with the failure of ipratropium bromide to decrease the amplitude of the diurnal variation in airway calibre or in bronchodilator responsiveness, do not support the use of ipratropium bromide as an additional treatment for children with asthma. The subjects in this study were clinically stable, and it is possible that a different result may have been found in children with unstable asthma.

The failure of ipratropium bromide to modify the amplitudes of the diurnal variations in airway calibre or in bronchodilator responsiveness suggests that these rhythms are not vagally mediated. Ipratropium bromide can cause vagal efferent blockade in man. ${ }^{1314} \mathrm{~A}$ dose of $40 \mu \mathrm{g}$ three times daily has been reported to cause bronchodilatation in children ${ }^{913} 14$ and to blunt the response to experimental bronchoconstriction. ${ }^{1314}$ The bronchodilator effect of ipratropium bromide peaks one hour after inhalation and virtually disappears within six hours. ${ }^{13}$ The fail ure of ipratropium bromide to diminish the diurnas variation in airway calibre and in bronchodilato responsiveness may be because its anticholinergie action does not last long enough to be effective in aif eight hourly regimen. The small but significan $\$$ increase in $P_{20}$ seen after treatment wity ipratropium bromide, despite an eight to 12 hour gap between the last dose of ipratropium bromide and the histamine challenge test, suggests, however, that the anticholinergic action of ipratropium bromide mas last longer than its detectable bronchodilator action?

\section{References}

1 Hetzel MR, Clark TJH Comparison of normal and asthmatic circadian rhythms in peak expiratory flow rate Thorax 1980;35:732-8.

2 Sly PD, Hibbert ME, Landau LI. Diurnal variation if peak expiratory flow rate in asthmatic children. Ped 60 atr Pulmonol 1986;2:141-6.

3 Johnston IDA, Anderson HR, Patel S. Variability of peak flow in wheezy children. Thorax 1984;39:593-

4 de Vries K, Goei JT, Body-Noord H, Orie NGMP Changes during 24 hours in the lung function and histamine hyperreactivity of the bronchial tree in astho matic and bronchitic patients. International Archives of Allergy 1962;20:93-101.

5 McGovern JP, Smolensky MH, Reinberg A. eds. Chrßె nobiology in allergy and immunology. Chicago, Illinois Charles C. Thomas, 1977.

6 Boushey HA, Holtzman MJ, Sheller JR, Nadel JA. Bron chial hyperreactivity. Am Rev Respir Dis 198. 121:389-413.

7 Phelan PD, Landau LI, Olinsky A. Respiratory illness children. 2nd ed. Oxford: Blackwell Scientific, 1982.3

8 Carswell F. Thirty deaths from asthma. Arch Dis Child 1985;60:25-8.

9 Mann NP, Hiller EJ. Ipratropium bromide in children with asthma. Thorax 1982:37:72-4.

10 Cox ID, Hughes DTD, McDonnell KA. Ipratropium bromide in patients with nocturnal asthma. Postgra Med $J$ 1984;60:526-8.

11 Yan K, Salome C, Woolcock AJ. Rapid method f@্ measurement of bronchial responsiveness. Thoras 1983;38:60-5.

12 Dunnett CW. Multiple comparison procedures for conf⿰亻弋 paring several treatments with control. Journal of tie American Statistical Association 1955;50:1096-121. 0

13 Pakes GE, Brogden RN, Heel RC, Speight TM, Ave GS. Ipratropium bromide: a review of its pharmat? cological properties and therapeutic efficacy in asthm? and chronic bronchitis. Drugs 1980; 20: 237-66.

14 Gross NJ, Skorodin MS. Anticholinergic, antimuscarina bronchodilators. Am Rev Respir Dis 1984;129:856-7\% 